\title{
tRNA fragments (tRFs) guide Ago to regulate gene expression post-transcriptionally in a Dicer-independent manner
}

\author{
CANAN KUSCU, ${ }^{1}$ PANKAJ KUMAR, ${ }^{1}$ MANJARI KIRAN, ZHANGLI SU, ASRAR MALIK, and ANINDYA DUTTA \\ Department of Biochemistry and Molecular Genetics, University of Virginia School of Medicine, Charlottesville, Virginia 22901, USA
}

\begin{abstract}
tRNA related RNA fragments (tRFs), also known as tRNA-derived RNAs (tdRNAs), are abundant small RNAs reported to be associated with Argonaute proteins, yet their function is unclear. We show that endogenous 18 nucleotide tRFs derived from the $3^{\prime}$ ends of tRNAs (tRF-3) post-transcriptionally repress genes in HEK293T cells in culture. tRF-3 levels increase upon parental tRNA overexpression. This represses target genes with a sequence complementary to the tRF-3 in the $3^{\prime}$ UTR. The tRF-3mediated repression is Dicer-independent, Argonaute-dependent, and the targets are recognized by sequence complementarity. Furthermore, tRF-3:target mRNA pairs in the RNA induced silencing complex associate with GW182 proteins, known to repress translation and promote the degradation of target mRNAs. RNA-seq demonstrates that endogenous target genes are specifically decreased upon tRF-3 induction. Therefore, Dicer-independent tRF-3s, generated upon tRNA overexpression, repress genes posttranscriptionally through an Argonaute-GW182 containing RISC via sequence matches with target mRNAs.
\end{abstract}

Keywords: tRNA fragments; tRF; post-transcriptional gene regulation; small noncoding RNA

\section{INTRODUCTION}

There are many different classes of cellular small RNAs, including microRNAs (miRNAs), Piwi-interacting RNAs (piRNAs) and tRNA related fragments (tRFs). tRFs are 14to 32-base-long RNAs derived from tRNAs that have been identified from bacteria to humans with high abundance. The $<26$ nucleotide (nt) long tRFs can be classified into four main groups: tRF-5s and tRF-3s, from the extreme $5^{\prime}$ and $3^{\prime}$ ends of mature tRNAs respectively; i-tRFs, internal fragments spanning anywhere in the mature tRNA but not mapping to the extreme $5^{\prime}$ or $3^{\prime}$ ends of mature tRNA; and tRF-1s, from the $3^{\prime}$ trailer of precursor tRNA (Lee et al. 2009; Haussecker et al. 2010; Telonis et al. 2015; for reviews, see Keam and Hutvagner 2015; Kumar et al. 2016). Several groups have developed bioinformatics tools to analyze tRNA-derived fragments from small RNA sequencing and generated several databases showing differential expression of tRFs in cell-lines, tissues and disease states (Kumar et al. 2015; Selitsky and Sethupathy 2015; Zheng et al. 2016; Pliatsika et al. 2018; Thompson et al. 2018).

The longer, 30- to 36-base-long fragments are also called tiRNAs/tRNA halves (tRHs) and have been tied to stress response (for review, see Saikia and Hatzoglou 2015). These $5^{\prime}$ tiRs/tRHs, produced by cleavage in the anticodon loop,

\footnotetext{
${ }^{1}$ These authors contributed equally to this work.

Corresponding author: ad8q@virginia.edu

Article is online at http://www.rnajournal.org/cgi/doi/10.1261/rna.066126. 118.
}

have essential roles in transgenerational gene regulation by metabolic stress: Mice with a high fat diet have higher levels of $5^{\prime}$ tiRs/tRHs in their sperm and these serve as signaling molecules in their offspring (Chen et al. 2016; Sharma et al. 2016).

As for tRF-3s, $<26$ base fragments from the $3^{\prime}$ end of mature tRNAs, several studies have suggested more diverse functions. Recently, Schorn and coworkers studied tRF-3s in mouse stem cells by comparing wild-type cells with Setdb1-/- cells lacking methylation on Histone 3 Lysine 9 (H3K9me3). They observed elevation of $18 \mathrm{nt}$ tRF-3a molecules and showed that they are necessary for the repression of LTR-retrotransposons (Schorn et al. 2017). Kim and coworkers identified a tRF-3 from LeuCAG3 tRNA (tRF-3011b), which they called LeuCAG3'tsRNA. This tRF is important for cell proliferation and enhances ribosome biogenesis by binding at least two ribosomal protein mRNAs, RPS28 and RPS15. They also showed that tRF-3011b is up-regulated in hepatocellular carcinoma and knockdown of tRF-3011b inhibits tumor growth (Kim et al. 2017). In addition, Maute and coworkers showed that a tRNA-derived miRNA (CU1276: tRF-3027b) is down-regulated in B-cell lymphoma. tRF3027b, $22 \mathrm{nt}$ in length, physically associates with Argonaute proteins and represses the expression of single strand DNA

(C) 2018 Kuscu et al. This article is distributed exclusively by the RNA Society for the first 12 months after the full-issue publication date (see http:// rnajournal.cshlp.org/site/misc/terms.xhtml). After 12 months, it is available under a Creative Commons License (Attribution-NonCommercial 4.0 International), as described at http://creativecommons.org/licenses/by-nc/4.0/. 
binding protein RPA1 in a sequence-dependent manner. Upregulation of RPA1, due to down-regulation of tRF-3027b in Burkitt lymphoma cell lines, results in aberrant proliferation of cells (Maute et al. 2013).

In addition to functions listed above, a meta-analysis of available short RNA sequencing data suggested that tRFs are present in mouse embryonic stem cells that are Dicer-/(Kumar et al. 2014). Moreover, analysis of PAR-CLIP data (Hafner et al. 2010) identified abundant tRF reads in Argonaute complexes, the main effector proteins in the miRNA pathway (Kumar et al. 2014). This analysis indicated that some tRF-3s and tRF-5s could bind to Ago proteins in a manner similar to miRNAs and use their $5^{\prime}$ seed sequence of 7-8 bases to bring their target mRNAs into the Ago complexes (Kumar et al. 2014). Most intriguingly, analysis of CLASH data from (Helwak et al. 2013) identified more tRF-3mRNA chimeras than miRNA-mRNA chimeras associated with Agol (Kumar et al. 2014). All these lines of evidence suggested a potential function of tRF-3s which interact with Argonaute proteins in regulating gene expression using a mechanism similar to miRNAs.

In this study, we focused on 18-nt tRF-3s which interact with Argonaute complexes. To experimentally test whether tRF-3s actually repress gene expression, we devised ways to up-regulate endogenous tRF-3 levels generated from tRNAs. We focused on three tRFs that were reported to be associated with Argonaute proteins in our previous report (Kumar et al. 2014). Overexpression of a particular tRNA (tRNA LeuAAG, tRNA CysGCA, or tRNA LeuTAA) resulted in production of the specific tRF-3 (tRF-3001, tRF-3003, and tRF-3009, respectively) (nomenclature based on tRFdb [Kumar et al. 2015]). The tRF-3s specifically down-regulate expression of luciferase reporters with a complementary sequence in the $3^{\prime}$ UTR. This repression is Dicer-independent but Argonaute-dependent, and also dependent on a match to the seed sequence at the $5^{\prime}$ end of the tRF. tRF-3:mRNA pairs associate with the "effector" GW182/TNRC6 proteins in the RISC, known to promote translational repression and target mRNA degradation. Furthermore, RNA-seq of HEK293T cells overexpressing the tRF-3009a showed that mRNA targets of tRF-3009a are significantly decreased, consistent with their degradation by the tRF-3s. Thus, tRF-3s, generated independently of miRNA biogenesis proteins such as Dicer and Drosha, are able to enter into Argonaute-GW182 containing RISC and regulate mRNAs post-transcriptionally via sequence complementarity.

\section{RESULTS}

\section{Overexpression of tRNA results in production of the corresponding tRF-3}

tRF-3s are 18-22 nt small RNAs of two distinct sizes: tRF-3a $(\sim 18 \mathrm{nt})$ and tRF-3b ( 22 nt) (Fig. 1A; Kumar et al. 2014). We focused on three tRF-3a that we knew to associate with AGO proteins in PAR-CLIP and CLASH data: tRF-3001a, tRF3003a, and tRF-3009a (Fig. 1B,C; Kumar et al. 2014). These three tRF-3s have very distinct lengths and locations on the parental tRNAs and are usually the most abundant fragments from the tRNA (Fig. 1D; Supplemental Fig. S1). They have been also detected in several different small RNA sequencing data sets (see tRFdb: http://genome.bioch.virginia.edu/trfdb/). We are aware of two technical issues that may limit the acquisition of a full repertoire of tRFs by small RNA sequencing. First, the many modifications of tRNAs or tRFs could block the reverse transcriptase producing smaller cDNA products than the actual RNA molecules. Second the size selection in many small RNA sequencing protocols may intentionally exclude small RNAs below or above a particular size. Despite these challenges, it is remarkable that small RNA sequence libraries from tens of laboratories yield tRFs of consistent size, sequence, and abundance.

We overexpressed the parental tRNAs in HEK293T cells and assessed tRNA and tRF levels by qRT-PCR. The overexpression of tRNA LeuAAG, tRNA CysGCA, and tRNA LeuTAA resulted in overproduction of the corresponding tRF-3s by sevenfold to 70-fold relative to endogenous tRF levels (Fig. 2A,B). Since PCR based detection of tRF-3s relies on reverse transcription (which could produce specific short cDNAs due to polymerase block by specific tRNA modifications), we also performed northern blot analysis to confirm the induction of tRF-3a and -3b in all three cases (Fig. 2C). To prove that tRF-3s produced by tRNA overexpression can be loaded into Argonaute, we performed Argonaute immunoprecipitation followed by northern blotting to show that tRF-3009a but not tRF-3009b is loaded into Argonaute (Fig. 2D; Supplemental Fig. S2).

\section{tRF-3s silence gene expression through sequence complementarity}

Luciferase reporters with perfect complementary sequences to the tRFs in the $3^{\prime}$ UTR were cotransfected with the tRNA. When tRF-3001 is expressed by overexpressing tRNALeuAAG and the luciferase $3^{\prime}$ UTR has a perfect complementary sequence to tRF-3001, the luciferase activity is repressed to $40 \%$ (Fig. 3A). Luciferase reporters with complementarity to the cognate tRFs were down-regulated similarly upon tRF3003 and tRF-3009 overexpression (Fig. 3A).

Titration of the amount of tRNA expressing plasmid showed that the down-regulation of luciferase is directly correlated with the amount of the tRNA expressing plasmid (Fig. 3B; Supplemental Fig. S3). We tested the specificity of the down-regulation by transfecting tRNA expressing plasmid and luciferase reporter plasmid with complementarity to the other two tRF-3s and no repression was observed (Fig. 3C). For example, tRF-3001 producing plasmid selectively repressed the luciferase reporter with complementarity to tRF-3001, but not those with complementarity to tRF-3003 or tRF-3009 and so on (Fig. 3C). To confirm that the repression 

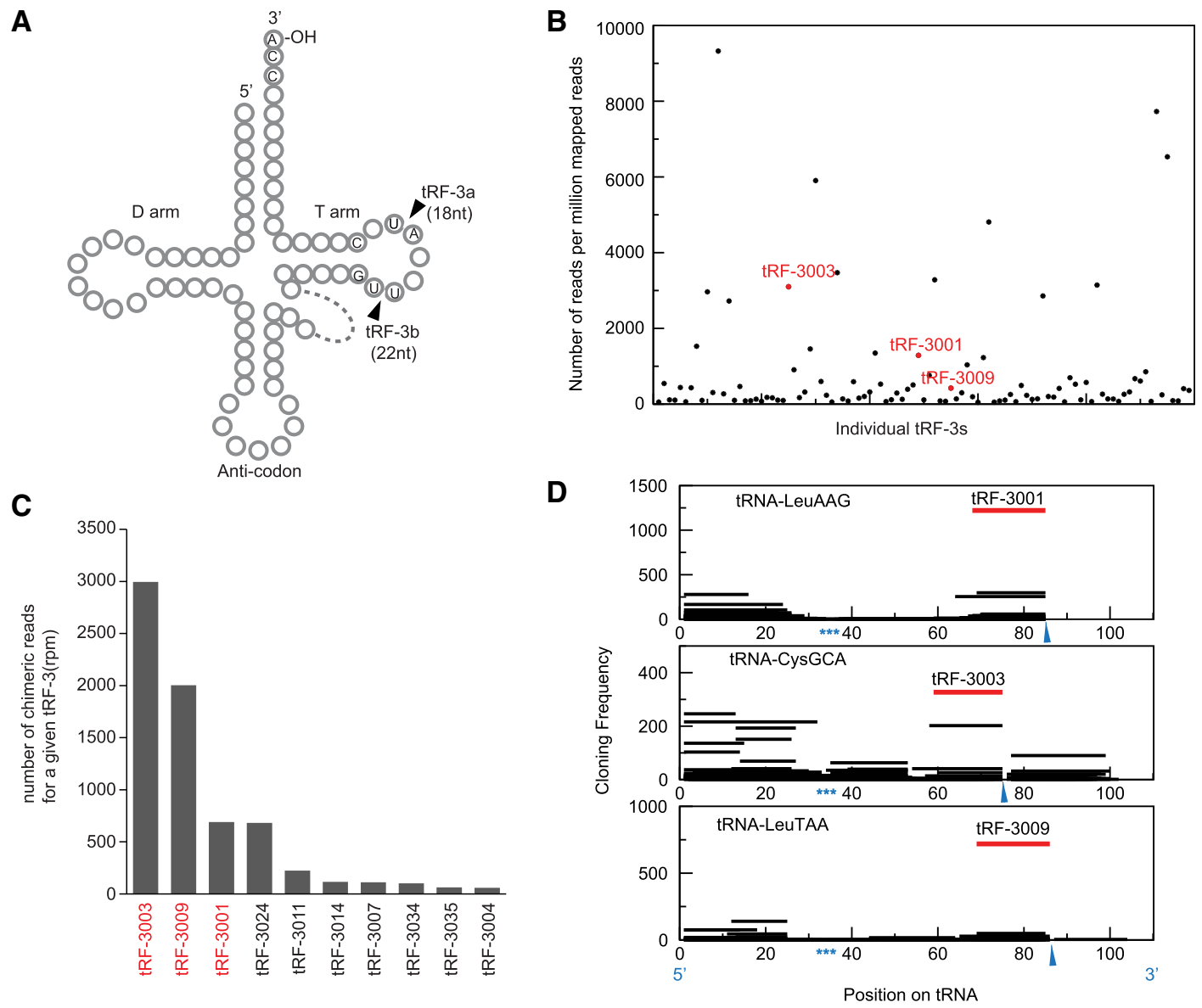

FIGURE 1. tRF-3s have distinct lengths and interact with Argonaute. (A) tRNA secondary structure depicting the tRF-3 cleavage sites. (B) Read counts for tRF-3s in AGO PAR-CLIP data (Hafner et al. 2010). Individual tRF-3s are arrayed along the $x$-axis with their expression levels (number of reads per million mapped reads) shown on the $y$-axis. The tRF-3s studied in this paper are indicated. $(C)$ Number of reads per million mapped reads for each tRF-3 in top 300 chimeric reads from Ago-CLASH data (Helwak et al. 2013). (D) Mapped position along the length of the parental tRNA ( $x$ axis) of small RNAs derived from that tRNA and their abundance ( $y$-axis: number of reads found in library GSM416733). Small RNAs from tRNA LeuAAG chr16.tRNA16 (upper), tRNA-CysGCA chr17.tRNA26 (middle), and tRNA LeuTAA chr6.tRNA83 (lower) are shown, and the tRFs studied in this paper indicated. $X$-axis is the position on tRNA gene. Blue arrowhead and $\left(^{* * *}\right)$ indicate the end of mature tRNA and anticodon, respectively.

observed in luciferase activity is mediated by tRF-3a and not due to other forms of tRFs, we transfected an 18-nt tRF3009a mimic and observed similar repression of a luciferase reporter with the "perfect comp" target site (Supplemental Fig. S4).

\section{tRF-3s repress targets with seed sequence matches}

Having identified the gene repression activity of tRF-3s, we wanted to characterize the base-pairing essential for target repression. We mutated the target site (tRF-complementary sequence) on the luciferase reporter to check whether disrupting certain base pairs between the tRF and the luciferase target would abolish luciferase repression. Note that microRNAs often recognize their target mRNAs by complementarity to a so called seed sequence at the $5^{\prime}$ end, which usually uses an A:T pair at the extreme $5^{\prime}$ end followed by 6-7 bases. In other words the mRNA target often pairs with the bases $1-7$ or bases $2-8$ at the $5^{\prime}$ end of the microRNA.

As mentioned above, tRF-3s can have two distinct lengths: $18 \mathrm{nt}$ (tRF-3a) or $22 \mathrm{nt}$ (tRF-3b), and the short RNA sequence data analysis suggests that tRF-3001 only has "a" form, while tRF-3003 and tRF-3009 are present in both "a" and "b" forms (Kumar et al. 2015). The luciferase reporters have perfect complementarity to the longer tRF-3b so that both forms of tRFs, if expressed, could repress the target. Mutations that disrupted pairing of the target with the $5^{\prime}$ seed of each tRF-3a diminishes repression (Fig. 4A: M1, M2, M3; Fig. 4B: M3, M4; Fig. 4C: M3, M4). The mutational analysis also showed that repression was mostly mediated via tRF-3a, because mutations at the extreme $3^{\prime}$ end of the target, that would disrupt pairing with the seed sequence of the longer tRF-3b, but not tRF-3a, continued to be repressed by the tRNA overexpression at almost the same level as the perfectly complementary target (Fig. 4B,C: M1, M2). 

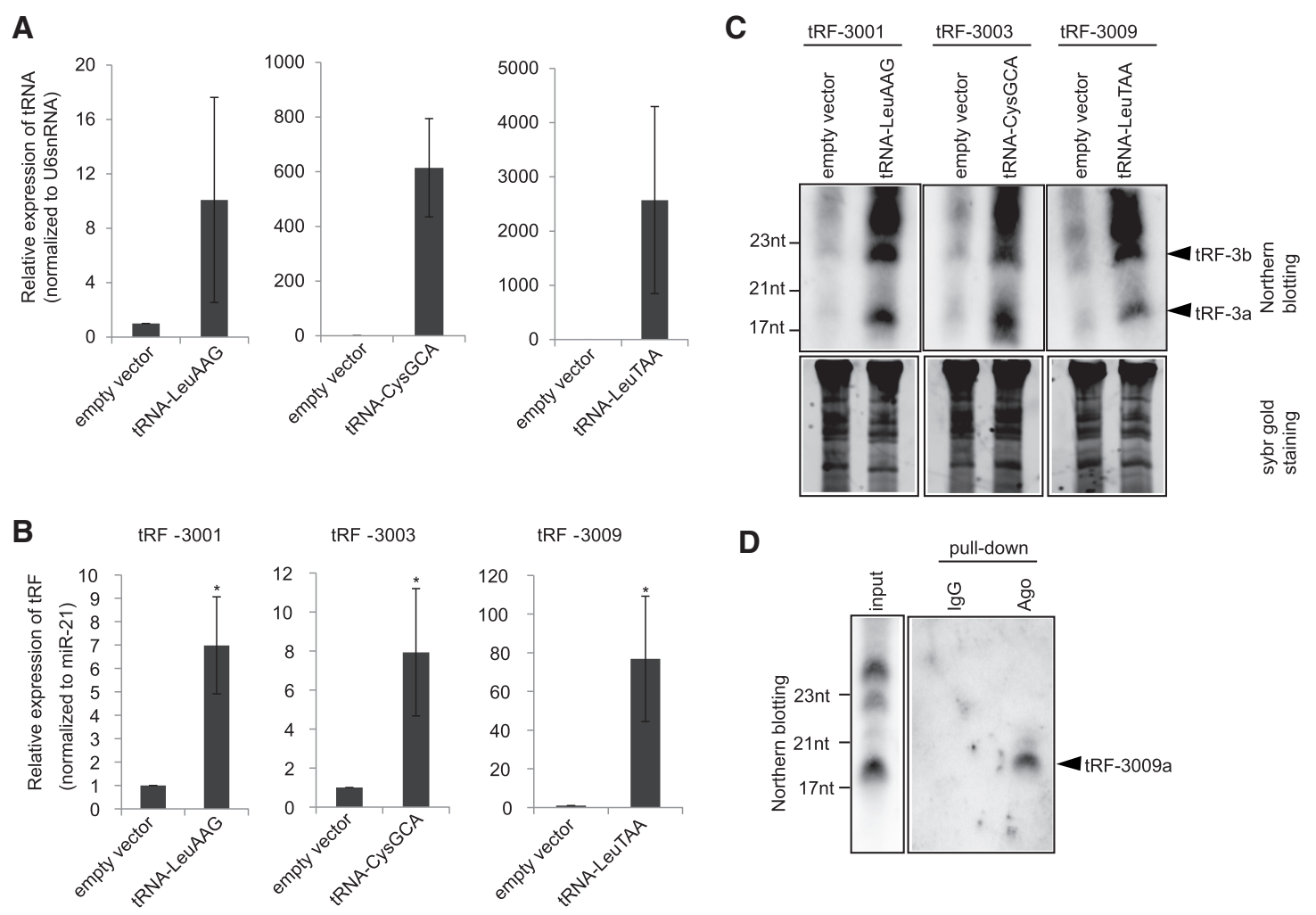

FIGURE 2. tRF-3s produced by tRNA overexpression are loaded into Argonaute. (A) Relative levels of indicated tRNAs upon tRNA overexpression. Mean and S.D. of three independent experiments. $\left({ }^{*}\right) P$-value $<0.05$ (Wilcoxon-Mann-Whitney Test). (B) Relative levels of indicated tRFs upon overexpression of the tRNAs indicated in $(A)$ in the same order from left to right. Mean and S.D. of at least three independent experiments. $\left({ }^{*}\right) P$-value $<0.05$ (Wilcoxon-Mann-Whitney Test). (C) Northern blot showing the tRF-3s produced after overexpression of the indicated parental tRNA. Lower panel shows equal loading of lanes. $(D)$ Northern blot showing the association with Argonaute of tRF-3009a produced from tRNA overexpression. The immunoblot showing successful Argonaute immunoprecipitation is in Supplemental Figure S2.

Perfect complementarity to the entire length of the tRF was not required for repression. For example, tRF-3003a can still repress a target that has mutations at the middle of the tRFmRNA pair indicating tolerance for a bulge structure in the middle of the tRF-mRNA pairing (Fig. 4B: M5). Mutations further away from canonical seed regions also allowed repression (Fig. 4A,B: M6). In all cases, however, mutations outside of canonical seed regions (Fig. 4A: M5; Fig. 4B,C: M5, M6, and M7) showed less repression than with the perfect match target, suggesting that tRFs appear to require additional complementarity outside the seed sequence for maximal repression. In parallel, we also performed luciferase assay with the reporter mutants using tRF-3009a mimic, which is a synthetic siRNA with the same sequence of tRF-3009a. The results were similar to what we observed with tRNA overexpression (Supplemental Fig. S4) confirming that the effects we have seen are due to tRF-3009a produced from tRNA LeuTAA. Thus, the rules of repression are similar to microRNAs, in that the target should be complementary to the $5^{\prime}$ seed sequence of the tRF. Though perfect complementarity to the entire length of the tRF is not essential for repression, additional complementarity downstream from the seed sequence facilitates repression.

\section{Gene silencing through tRF-3 is dependent on Argonaute proteins}

The seed sequence properties of tRF-3 (Fig. 4) is reminiscent of microRNA rules and consistent with our hypothesis that tRF-3 can enter Ago complexes to perform microRNAlike functions. Our previous analysis of AGO PAR-CLIP showed that tRF-3s associate with Argonaute proteins (Fig. 1B; Kumar et al. 2014). Moreover, AGO1 CLASH analysis revealed that tRF-3s form chimeras with mRNAs in AGO1 (Fig. 1C; Kumar et al. 2014). We have also demonstrated by northern blot that tRF-3009a produced from tRNALeuTAA overexpression is loaded into Argonaute (Fig. 2D). To test whether Argonaute proteins (Ago) are essential for the gene repression function of tRF-3s, we performed the luciferase reporter assays upon Ago-1, -2, and -3 knockdown by siRNA. Ago-4 levels also decreased upon knockdown of Ago1, 2, and 3 (Supplemental Fig. S5A). As seen in Figure 5A, the repression of the luciferase reporter by tRF-3009 was diminished when the Argonaute proteins were down-regulated. Repression by tRF-3001 or tRF-3003 was also attenuated in response to lower Argonaute protein levels (Supplemental Fig. S5B,C). 
A

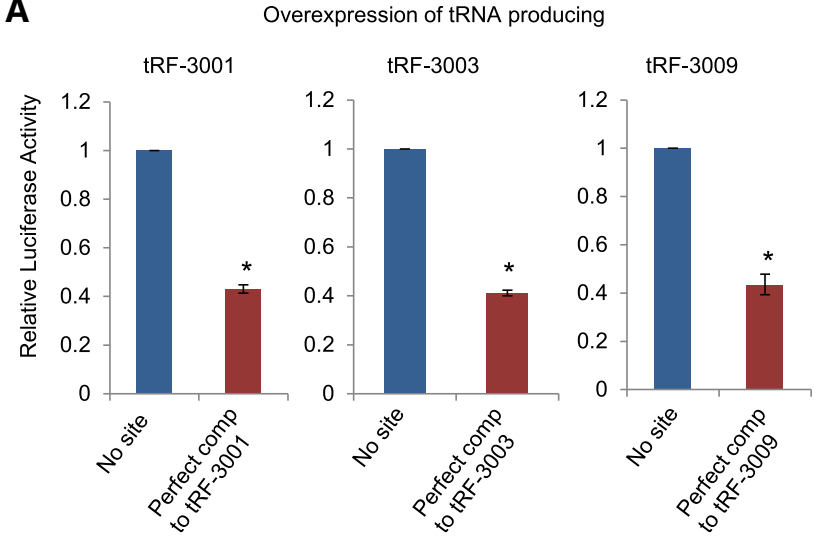

B

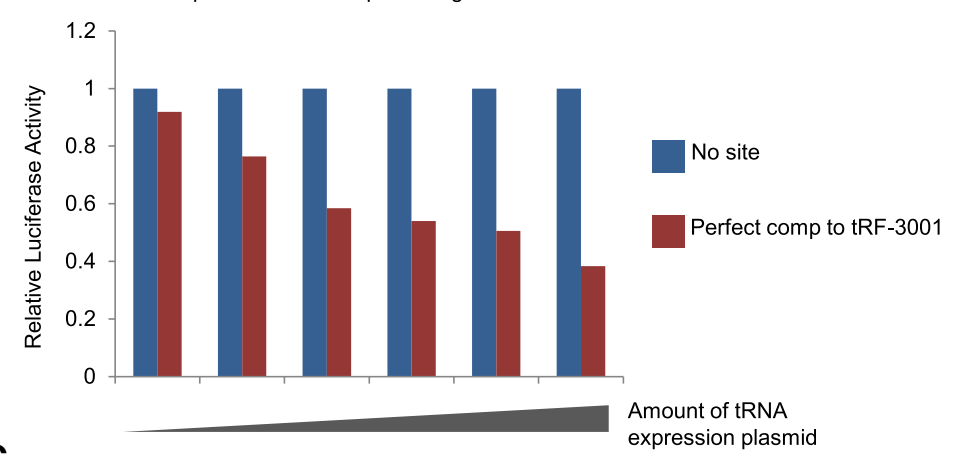

C

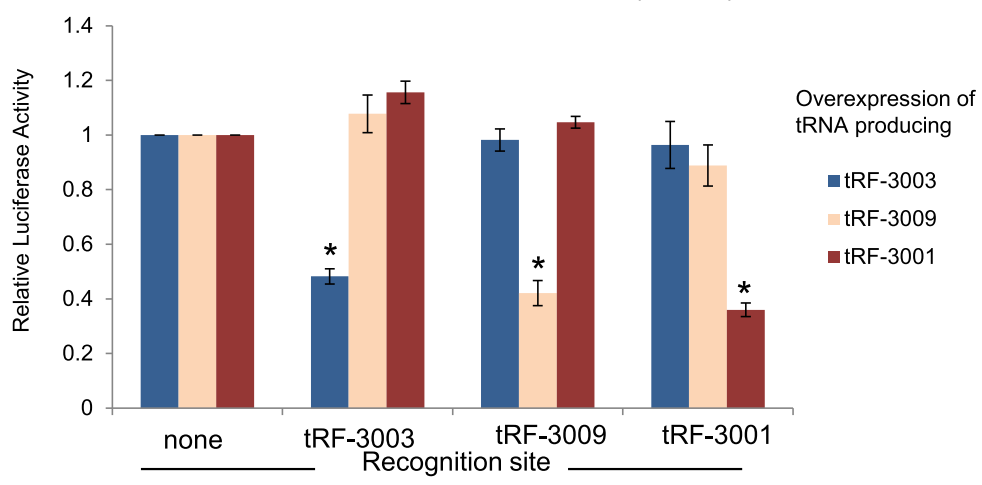

FIGURE 3. tRF-3s down-regulate target expression through complementarity in $3^{\prime}$ UTR of luciferase reporter. $(A)$ Luciferase reporter assays using Renilla luciferase with a perfect complementary sequence to tRF-3 at the $3^{\prime}$ UTR. Renilla luciferase levels were first normalized to Firefly luciferase levels from the same transfection and then normalized to no tRNA/tRF overexpression (empty vector) control. (*) $P$-value $<0.05$ ( $t$-test). (B) Degree of repression correlates with tRNA overexpression amount. Amount of transfected tRNA plasmid was titrated to measure the change in the degree of repression. Luciferase reporter assays were analyzed as described in $A$. $(C)$ Luciferase reporter assay showing the specific repression by each corresponding tRF-3. (*) $P$-value $<0.05$ ( $t$-test).

Catalysis by Argonaute may generate Dicer-independent miRNA, miR-451 (Cheloufi et al. 2010). We therefore checked the levels of tRF-3s in Ago knockdown conditions since loss of repression seen in Figure 5A might be due to a defect in the biogenesis of tRF. By northern blot analysis, tRF-3009 is still generated as efficiently as in WT cells after Ago knockdown (Fig. 5B). Therefore, Ago is necessary for gene repression by tRF-3s but not for their biogenesis.

\section{Biogenesis of tRF-3 is independent of Dicer, Drosha, and Exportin 5}

One of the major enzymes in miRNA pathway is Dicer, which cuts out the loop in the stem-loop hairpin structure of the precursor miRNA (pre-miRNA) to generate miRNA (Bernstein et al. 2001). The role of Ago in tRF-3 mediated repression prompted us to check whether Dicer is important for tRF-3 mediated repression. Dicer could have a role in loading tRF-3 into Ago complexes, or in tRF-3 biogenesis. We obtained Dicer knockout 293T cell lines that have been shown to be defective in repressing microRNA targeted luciferase reporters (Bogerd et al. 2014). Analysis of small RNA sequencing data from these cells showed that although Dicer is required for microRNA biogenesis, it is not required for the biogenesis of tRF-3s including tRF-3001, tRF-3003, or tRF-3009 (Fig. 5C; Supplemental Fig. S6B). Furthermore, the luciferase repression by tRNA generated tRF-3 is still observed in the Dicer knockout cells, and is even more pronounced than in wild-type cells (Fig. 5D), most probably because more Argonaute protein becomes available due to the failure to produce cellular miRNA. Therefore, Dicer is not required to produce the 18-nt tRF-3a nor is it essential for tRF-3 loading into Ago.

Our previous report showing that tRF abundance was unchanged in the absence of Dicer or DGCR8 was derived from embryonic stem cells (Kumar et al. 2014). Besides the Dicer knockout 293T cells described above, Dicer-/-, Drosha-/-, and Exportin 5-/- HCT116 colon cancer cells have been generated using CRISPR-Cas9 technology, providing a second opportunity to test the importance of these enzymes in tRF biogenesis (Kim et al. 2016). Analysis of the small RNA sequences from these cells showed that although Dicer and Drosha are important for microRNA levels, they are dispensable for tRF-3 generation (Supplemental Fig. S6C,D). Exportin 5 depletion did not affect tRF-3 levels (Supplemental Fig. S6D), similar to previous observations that Exportin 5 is dispensable for miRNA biogenesis (Supplemental Fig. S6C; Kim et al. 2016). tRF-3016 is an exception in that it is significantly repressed in the Exportin 5 deleted cells due to unknown reasons. 


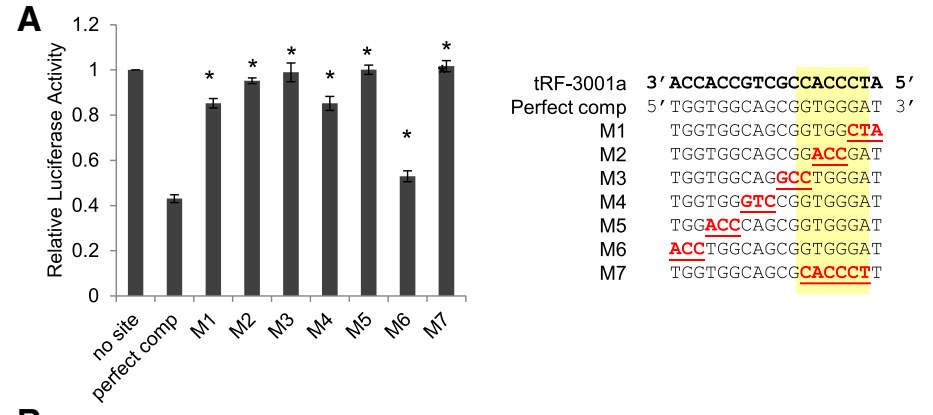

B

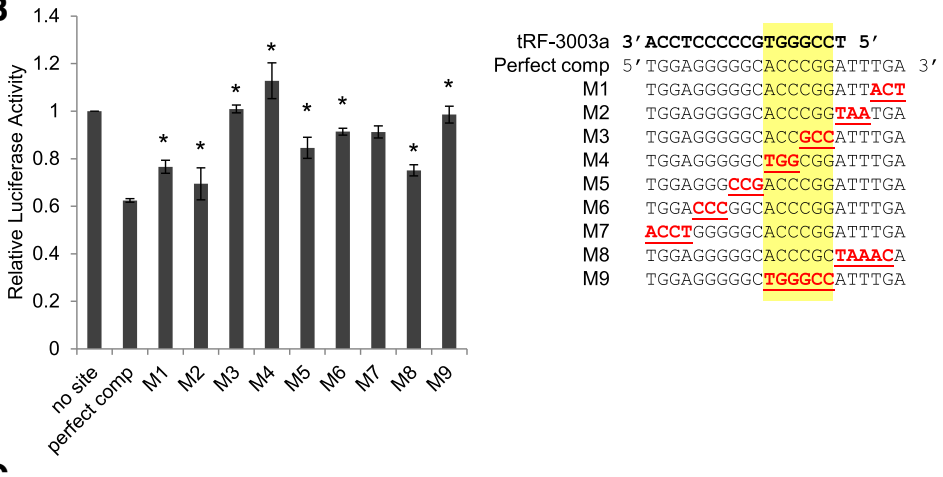

C

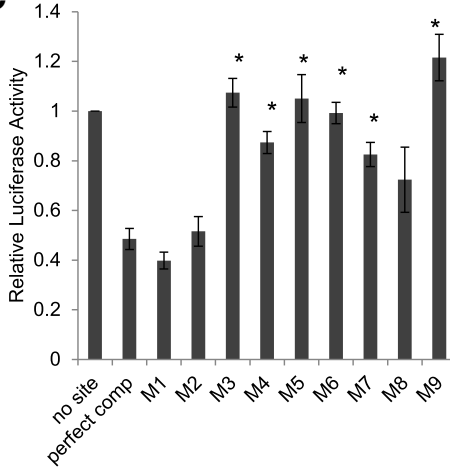

FIGURE 4. Seed sequence is required for target repression by tRF-3s. Luciferase reporter assays with mutant target site at the luciferase reporter upon tRF-3001 (A), tRF-3003 (B), and tRF-3009 $(C)$ overexpression. Canonical seed region on each tRF-3 and complementary sequence on each target are highlighted in yellow. Mutated regions are underlined and colored red. $P$-values are calculated by $t$-test comparing luciferase reporter with indicated target sequence to empty vector control $\left({ }^{*}\right) P$-value $<0.05 ;(* *) P$-value $<0.005$ ( $t$-test).

\section{tRF-3s interact with GW182/TNRC6A, the effector protein for translational repression and mRNA degradation}

In addition to Dicer and TRBP, AGO interacts with GW182 proteins in RISC complex. GW182 interaction with AGO is necessary for translation repression and degradation of target mRNA (Eulalio et al. 2008). PAR-CLIP of GW182 showed that it interacts with miRNAs and mRNA targets (Hafner et al. 2010). To test whether tRF-3s also interact with GW182 proteins, we investigated the association of tRF-3s with GW182 (TNRC6A) and its paralogs (TNRC6B and C) by analyzing PAR-CLIP data from HEK293 cells (Hafner et al. 2010). As seen in Figure 6A, TNRC6A/B/C associate with some tRF-3s, but not all. The most abundant association was observed for TNRC6A, in which case a few tRF-3s are present at levels comparable to miRNA levels (>1000 RPM), although in general tRF-3s associate with TNRC6 proteins at a lower level compared to microRNAs. Moreover, the locations of the $\mathrm{T}$ to $\mathrm{C}$ mutations in the PAR-CLIP data indicate that tRF-3s directly interact with TNRC6 proteins at position 12 and 14, while sparing bases 1-6 that overlap with the seed (Fig. $6 \mathrm{~B})$. This interaction is reminiscent of miRNA-TNRC6 interaction (Hafner et al. 2010), at position 11 and 13, with the bases 1-6 in the seed sequence being spared from the cross-link.

The absence of any cross-link of the TNRC6 with the seed sequence of the tRF-3 or microRNA is likely because the seed is paired with the target RNA even in the TNRC6 complexes. Since GW182/TNRC6A associates with AGO in the RISC, we wondered whether the targets of the tRF-3 may also be detected in the TNRC6 PAR-CLIP data. We therefore searched in the PAR-CLIP data for target RNA reads with complementarity either to seeds of microRNAs, or specifically to tRF-3s but not microRNAs. Targets that were detected were aligned with the $\mathrm{T}$ to $\mathrm{C}$ mutation marking the cross-link at the center along with 20 bases up and downstream from the cross-link to produce cross-link-centered-RNAs (CCRs). Target RNAs complementary to microRNAs can be detected in the CCRs associated with TNRC6 (Fig. 6C). Most interesting, target RNAs with complementarity to tRF-3 seeds (1-7 mer or 28 mer) are also associated with the TNRC6 (Fig. 6D). In the case of both the microRNAs and the tRF-3s, the most frequent complementarity to the seed is seen just downstream from the cross-link center, similar to what is seen with the microRNA:target or tRF-3:target pairs in Ago1-4 PAR-CLIP data (Hafner et al. 2010). This result is consistent with the proposal that, as with microRNAs, GW182 associates with the tRF3:target in the Ago containing RISC to promote translation repression and target mRNA degradation.

\section{RNA-seq analysis reveals that $t R F-3009 a$ represses endogenous mRNA targets}

GW182 associated with miRNA-mRNA loaded RISC complex mediates the degradation of mRNA targets (for review, 
A

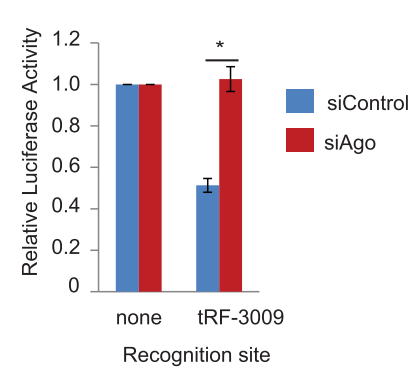

B

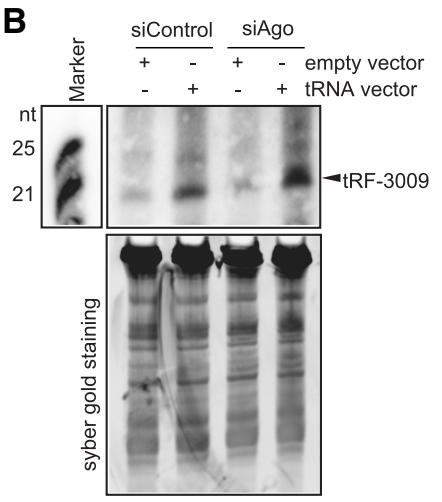

D

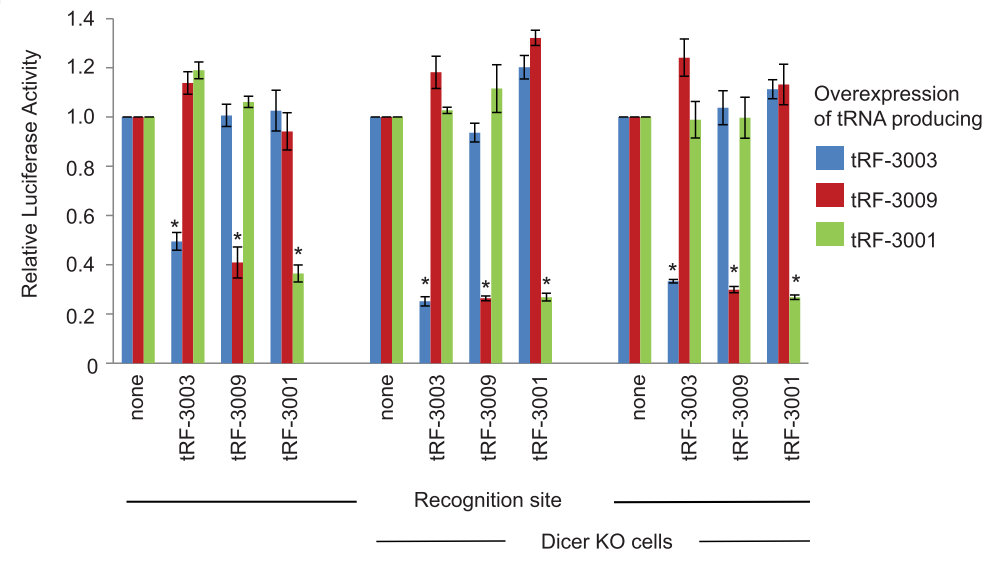

C

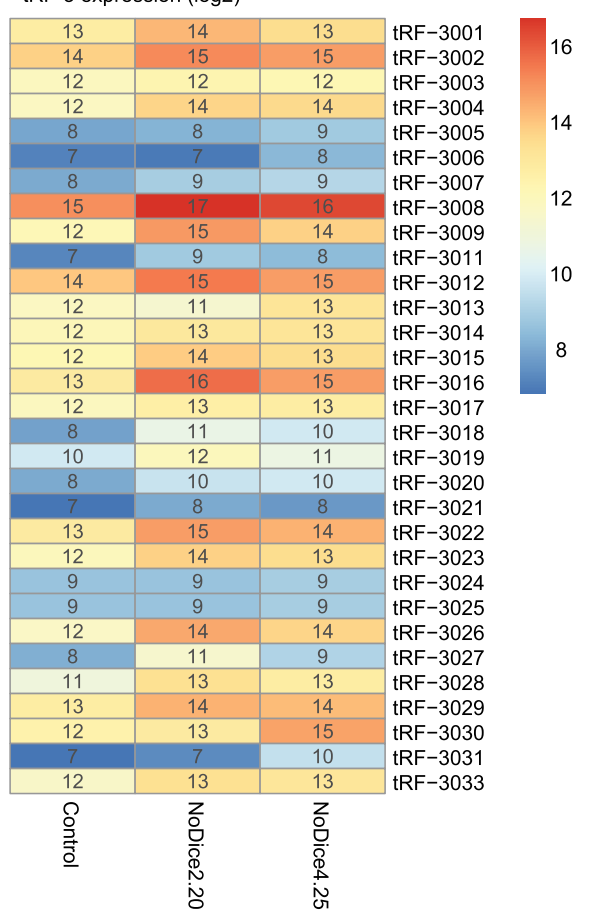

FIGURE 5. Target repression by tRF-3s is independent of Dicer but dependent on Argonautes. (A) Luciferase reporter assays after tRNA overexpression to produce tRF-3009 \pm knockdown of Argonaute proteins. $\left(^{*}\right) P$-value $<0.05$ ( $t$-test). $(B)$ Northern blot showing tRF-3009 levels in tRNA overexpressing cells after Ago knockdown. Lower panel shows equal loading of lanes. (C) tRF-3 read counts in small RNA sequencing data from WT and two different Dicer knockout clones of HEK293T. Small RNA sequencing data from Bogerd et al. (2014) (D) Luciferase reporter assays in wild-type and Dicer knockout HEK293T cells; NoDice 4-25 (middle), and NoDice 2-20 (right) (Bogerd et al. 2014).

see Jonas and Izaurralde 2015). Since tRF-3s paired with their target RNAs are also found associated with GW182 proteins, we first checked the mRNA levels of luciferase in luciferase reporters containing a perfect complementary site to tRF3009. In this experiment, we assayed both the luciferase protein level by luciferase assay and the luciferase mRNA level by qRT-PCR from the same cells. As seen in Figure 7A, mRNA and protein levels of Renilla luciferase are both down-regulated to $\sim 50 \%$. This suggests the tRF-3 mediated repression occurs mostly by degrading the target RNA, most likely by the de-adenylation/de-capping followed by exonuclease digestion that has been proposed for microRNA targets.

Next, we analyzed cellular mRNA expression changes by RNA-sequencing after tRNA overexpression mediated tRF overproduction. Overexpression of tRNA Leu TAA, which produces tRF-3009a, repressed target mRNAs with $3^{\prime}$ UTRs bearing complementarity to at least the 6-mer seed sequence of the tRF, as predicted by RNA22 (Miranda et al. 2006), relative to RNAs that do not have such complementarity (Fig. 7B). This experiment was repeated again and cumulative distribution function $(\mathrm{CDF})$ plots of the second replicate shows a similar trend (Supplemental Fig. S7).

The top five repressed targets from RNA-seq contain not only base complementarity to the tRF-3009a seed but also have some complementarity downstream from the seed (Fig. 7C). We first validated the decrease in RNA of the top five repressed targets by qRT-PCR (Fig. 7D). Moreover, we cloned the $3^{\prime}$ UTR of these targets into luciferase reporter plasmids and performed luciferase reporter experiments. Luciferase reporters containing the $3^{\prime}$ UTRs of all these genes are repressed upon overexpression of tRNA-LeuTAA producing tRF-3009s (Fig. 7E). This result independently validates the repression observed in the RNA-seq experiment.

\section{DISCUSSION}

tRNA-derived RNA fragments (tRFs) have been discovered and characterized from small RNA sequencing, and so certain technical limitations should be recognized. First, base modifications in tRNAs and tRFs could block the progression of the reverse-transcriptase, leading to artificially truncated cDNA products. To guard against this, key results in this paper have been validated by northern blotting for tRFs of the correct size. Second, many of the cloning protocols will not clone fragments that lack $5^{\prime}$ phosphate or have a $2^{\prime}-3^{\prime}$ cyclic phosphate at the end (as with $5^{\prime}$ tiRs/tRHs). Last, size selection, often used during microRNA sequencing, can artificially limit the recovery of tRNA fragments below or above a certain size. Because of these limitations, small RNA sequencing data needs to be analyzed while paying attention to these technical differences, as 


\section{A}
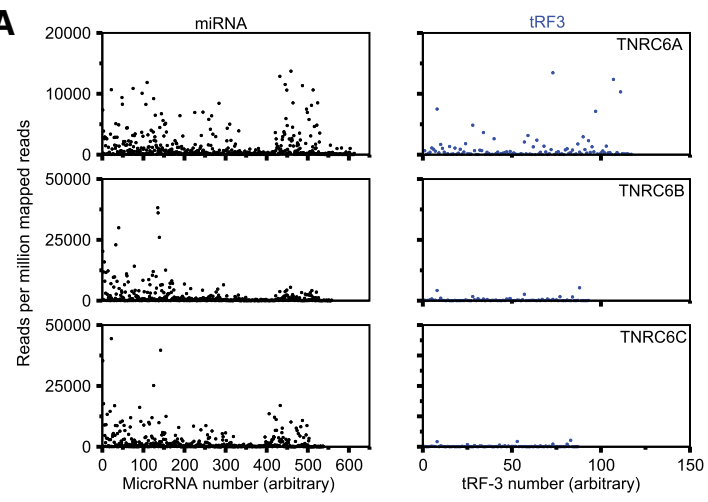

B
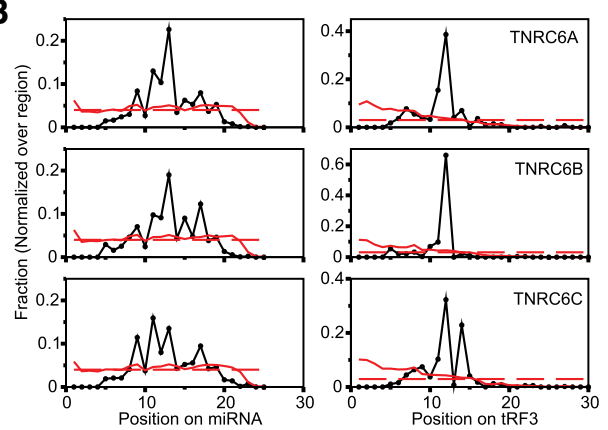

C

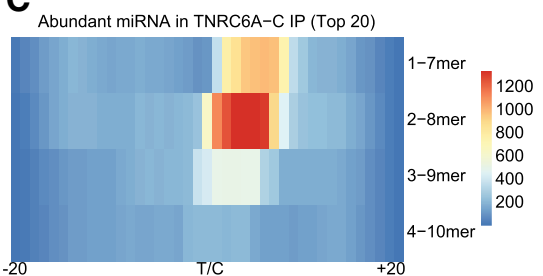

D

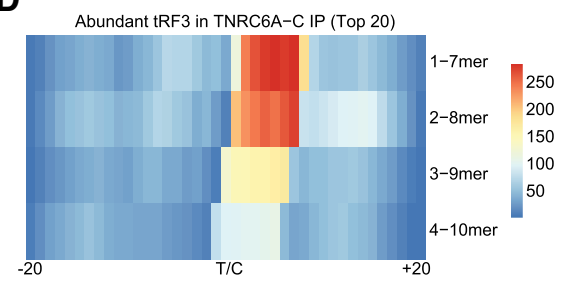

FIGURE 6. tRF-3s associate with GW182/TNRC6 proteins. (A) Read counts for miRNAs or tRF$3 \mathrm{~s}$ in TNRC6A, TNRC6B, and TNRC6C PAR-CLIP data from Hafner et al. (2010). Each microRNA or tRF is given an arbitrary identifying number along the $x$-axis. The expression level (expressed as reads per million mapped reads) of each microRNA or tRF is shown on the $y$-axis. (B) Normalized positional T to C mutation frequencies for miRNA or tRF-3 reads found in the TNRC6A, TNRC6B, and TNRC6C PAR-CLIP data. $(C, D)$ Complementarity in the target RNA cross-link-centered RNAs (CCRs) present in the TNRC6A PAR-CLIP to the 1-7, 2-8, 3-9, and $4-10$-mer sequences from the $5^{\prime}$ end of the 20 most abundant microRNAs or tRF-3s seen in $B$. The CCRs are centered on the site of the U/C mutation and the number of targets with complementarity indicated at the corresponding base in the sequence of the CCR. For example, the maximum number of matches is seen with the 1-7 mer of the tRF and begins with the base immediately downstream from the cross-link site in the target RNA.

we have done here. In this paper we focus on tRF-3s detected in all small RNA sequencing libraries, present at a reasonable abundance relative to other fragments and shown to interact with Argonaute proteins. Undoubtedly new tRFs will emerge and the abundance of tRFs will increase as new techniques are deployed to sequence tRNAs and tRFs that overcome limitations due to tRNA modifications (Cozen et al. 2015; Zheng et al. 2015). In particular, cP-RNA-seq, developed to selectively amplify and sequence RNAs that end in cyclic phosphates, will increase the recovery of $5^{\prime}$ tiRs/tRHs (Honda et al. 2016). These new tRFs may have completely different functions and may not act in the manner of the tRF-3 we study here.

\section{tRF-3 targets}

miRNAs interact with their target mRNAs by base-pairing. In plants, perfect complementarity is essential for miRNA-mRNA targeting. In metazoans, with few exceptions, miRNA-mRNA base-pairing occurs imperfectly allowing unmatched bulges between the two RNAs. The miRNA-mRNA base-pairing rules are defined based on both bioinformatics and experimental results. The most important rule is that miRNAs recognize their mRNA targets using their 27 (or 8) nt sequence at their $5^{\prime}$ ends, which is defined as the seed sequence. Second, an unpaired region at the middle of miRNA-mRNA pairing (bulge) is tolerated. Third, following the bulge there is often sequence complementarity between miRNA and mRNA (Brennecke et al. 2005; Lewis et al. 2005; Grimson et al. 2007; Nielsen et al. 2007; and reviewed in Filipowicz et al. 2008). We show that tRF-3s down-regulate target gene expression in a sequence-dependent manner similar to miRNAs. Moreover, tRF-3: mRNA pairs present in the Ago containing RISC also interact with GW proteins, mainly TNRC6A. Importantly, our qRTPCR analysis on luciferase reporters and RNA-seq analysis on endogenous genes show that tRF-3s repress mRNA levels.

\section{The roles of Drosha, Dicer, and Argonaute proteins}

The analysis of publicly available short RNA sequences from Dicer knockout mouse embryonic stem cells showed that Dicer is not required for generation of tRF-3s (Kumar et al. 2014, 2015). In contrast, there are papers reporting that Dicer might be necessary for tRF generation (Cole et al. 2009; Maute et al. 2013). It has been suggested that tRNA may fold in alternate structure and that the reported role of Dicer in tRF generation is due to the alternate structure that makes the tRNA susceptible to Dicer (Schopman et al. 2010). We find, however, that in multiple cell lines tRF-3 generation is Drosha- and Dicerindependent and yet Argonaute proteins are essential for their function (Fig. 5; Supplemental Fig. S6). Remarkably, tRF-3s repress their targets better in Dicer knockout cells suggesting that Argonaute proteins are more accessible for tRF-3 loading in the absence of miRNAs (Fig. 5D). This result raises the possibility that $\mathrm{tRF}$ become even more important 
A
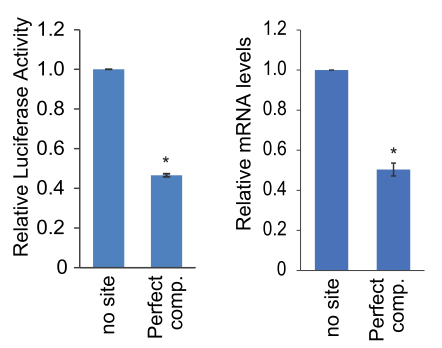

C

5' UGAAUGAAUAUAGUGGGGG 3' FER1, 3'UTR-8305

3' AC-CAUGGUCCUCACCCCA 5' tRF-3009a

5' AUGGAGUAGGAGUGGGGC 3' FER1, 3'UTR-8697

3' ACCAUGGUCCUCACCCCA 5' tRF-3009a

5' GAAGCUCUGGAGUGGGGC 3' DGCR2, 3'UTR-761

3' ACCAUGGUCCUCACCCCA 5' tRF-3009a

D

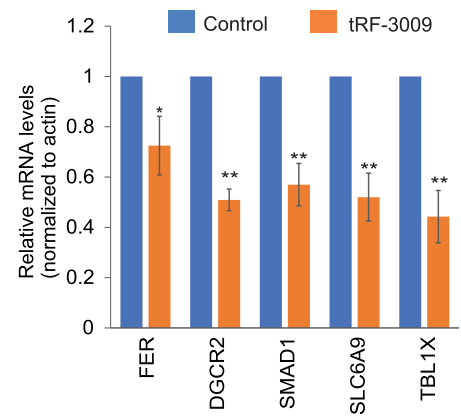

B

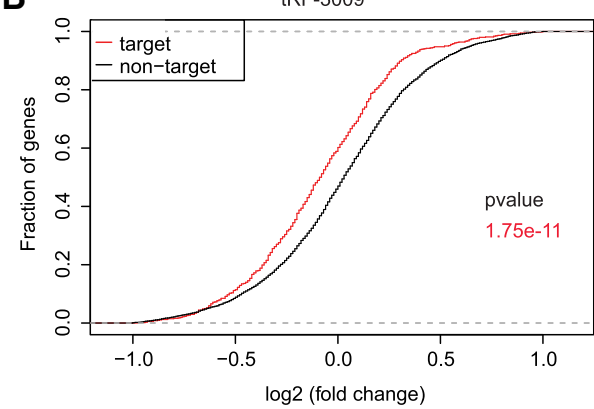

5' UUG-AGGUGG-GUGGGGG 3' SMAD1, 3'UTR-658 3' ACCAUGGUCCUCACCCCA 5' tRF-3009a

5' UGGG-GGAUGGGGUGGGGG 3' SLC6A9, 3'UTR-693 3' A-CCAUGGUCCUCACCCCA 5' tRF-3009a

5' UGGC-CCAGCCCGUGGGGG 3' TBL1X, 3'UTR-1865 3' ACCAUGGUC-CUCACCCCA 5' tRF-3009a

$\mathbf{E}$

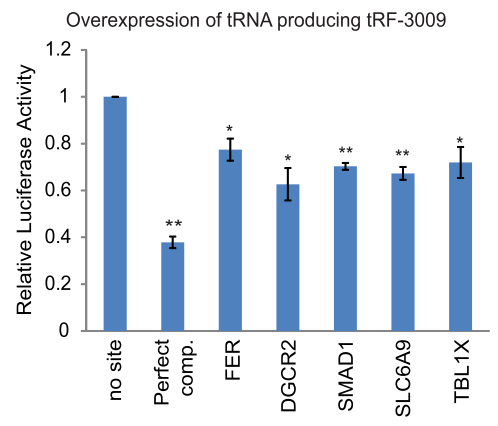

FIGURE 7. Endogenous targets are repressed upon tRF-3009 overexpresssion by tRNA-LeuTAA transfection. (A) Luciferase assay upon tRNA overexpression to produce tRF-3009. In parallel, Renilla luciferase mRNA levels were measured by qRT-PCR from the same cells on which luciferase assays were performed (normalized to Firefly mRNA levels) $\left(^{*}\right) P$-value $<0.005$. (B) Cumulative distribution function (CDF) plots showing the repression of tRF-3009 targets upon overexpression of tRNA producing tRF-3009. Targets have been predicted using RNA22 algorithm (Miranda et al. 2006). (C) Seed sequence complementarity in selected tRF-3009 targets that are identified in RNA-seq upon tRF-3009 expression. The number after 3' UTR indicates the start position of the mRNA sequence match with 1 being the base immediately downstream from the stop codon. (Red line) Perfect base-pairing in seed; (black line) perfect base-pairing outside seed; (dashed line) wobble base-pairing. FER1 gene has two predicted complementary sites on its $3^{\prime}$ UTR. (D) Relative mRNA levels of indicated genes upon tRNA-LeuTAA transfection, leading to tRF-3009 overexpression. $\left(^{*}\right) P$-value $<0.05$; $\left(^{* *}\right) P$-value, 0.005 ( $t$-test). (E) Dual luciferase reporter assay on reporters containing the $3^{\prime}$ UTR of indicated genes after tRNA-LeuTAA transfection, leading to tRF-3009 overexpression. Perfect complementary sequence to the tRF-3009 serves as a positive control and all results are normalized to the "no site" reporter without any match to the tRF-3009 (*) $P$-value $<0.05$; $(* *) P$-value, 0.005 ( $t$-test).

regulators of gene expression in the absence of miRNAs. Down-regulation of Dicer and/or Drosha have been correlated with a worse outcome in lung, breast, skin, endometrial, and ovarian cancer (for review, see Foulkes et al. 2014). Our results suggest that tRF-3s acquire more potency when there are lower levels of Dicer or Drosha so that we should investigate whether tRFs are important for the poorer outcome in these tumors.

On the other hand, Dicer has an important role in loading microRNAs into Argonaute complexes (Chendrimada et al. 2005; Gregory et al. 2005). The fact that tRF-3s can repress

genes by a mechanism dependent on Argonaute proteins in Dicer knockout cells suggests that Dicer is not essential for loading of tRF-3s into Argonaute. It is still possible that the Dicer-independent loading of tRF-3s into Argonaute is less efficient than the Dicer-dependent loading of microRNAs. However, our results open the possibility that other short RNAs, if present at high concentration, may load on to Argonaute complexes and repress gene expression. Similarly, transfected miRNAs can load into RISC and repress their targets in the absence of Dicer in mammalian cells (Betancur and Tomari 2012). Hsp90 proteins help the stability of unloaded Argonaute proteins and loading of miRNA to Argonaute in an ATP-dependent way (for review, see Meister 2013), and thus Hsp90 may be involved in the loading of tRFs on Argonaute proteins by this Dicer-independent pathway. It is worth noting that agotrons, short introns interacting with Argonaute proteins, were shown to be loaded into Argonaute independently from Dicer, suggesting that such loading is not unique to tRFs (Hansen et al. 2016).

\section{Regulators of tRF-3 levels}

The lack of any requirement for Dicer or Drosha for tRF-3 production opens up the question of how tRF-3s are generated. Identification of the specific enzyme/enzymes that are important for tRF generation will let us better understand tRF biology and function. The tRF-3s that we studied here contain CCA sequence which indicates that they are generated from mature tRNA. We hypothesize that any process or enzyme regulating tRNA synthesis, maturation, or charging, such as levels/activities of CCA addition enzyme, tRNA modifiers like tRNA methlytransferases or aminoacyl-tRNA synthetases will affect the levels of tRF-3s. Indeed, multiple tRNAs have been reported to be up-regulated in breast cancer (PavonEternod et al. 2009). Recently, Goodarzi et al. identified two up-regulated tRNAs which result in overexpression of prometastatic proteins in metastatic breast cancer cells. Whether tRFs derived from these tRNAs are up-regulated in parallel to control gene expression and contribute to the phenotype should be investigated. Moreover, the Myc oncogene was shown to up-regulate Pol III transcripts including tRNAs 
(Gomez-Roman et al. 2003; Arabi et al. 2005; Grandori et al. 2005; Lin et al. 2012). Since overexpression of tRNA in this paper resulted in over production of tRF-3s, it is possible that when tRNA expression is induced by c-Myc, there will be more tRF-3s. In parallel, many other oncogenes are known to induce tRNAs. Examples include Ras, Raf, EGF receptor and oncogenes like E6 and E7 from human papilloma virus (that inhibit p53 and $\mathrm{Rb}$, both known to repress tRNA transcription) (Grewal 2015). Thus, it is interesting to speculate that these oncogenes may indirectly increase the levels of specific tRFs. In line with this, there is growing literature that levels of tRNA-derived fragments are significantly altered in many cancers (Zheng et al. 2016). More work is clearly needed to establish (a) whether tRF levels are systematically altered in cancers and (b) whether these small tRFs can regulate gene expression and the phenotypes of the cancers.

As more tRFs are implicated in different functions, it is exciting to note that at least a sub-fraction of the tRFs also regulate gene expression by utilizing Argonaute-GW182 mediated pathways that were first discovered in the context of microRNAs.

\section{MATERIALS AND METHODS}

\section{Cloning}

tRNA genes including their upstream and downstream elements which presumably regulate their expression levels were amplified with specific primers and cloned by infusion cloning into pcDNA3 vector (see Supplemental Table 1 for primers and Supplemental Table 2 for list of plasmids). The genomic locations that are used for the expression of tRNAs are as follows: tRNA LeuAAG chr16.tRNA16 chr16:22308161-22308710 (hg19), tRNA-CysGCA chr17.tRNA26: chr17:37310553-37311015 (hg19), and tRNA LeuTAA chr6.tRNA83 chr6:144537474-144537897 (hg19).

Synthetic oligonucleotides containing perfect complementarity to tRF-3s were cloned at the $3^{\prime}$ UTR of the Renilla luciferase gene in psi-CHECK2 (Promega) reporter plasmid by infusion cloning between PmeI and XhoI sites for luciferase assays. 3'UTRs of endogenous genes were similarly cloned into psi-CHECK2 (Supplemental Table 2).

\section{qRT-PCR analysis of tRNAs/tRF-3s}

Four micrograms of tRNA overexpression plasmids were transfected into HEK293T cells in $6 \mathrm{~cm}$ plates using Lipofectamine 2000 (Thermofisher). The cells were collected after $2 \mathrm{~d}$ of transfection and total RNA was purified for further analysis.

For detection of tRNAs by qRT-PCR, total RNAs were purified with TRIzol (Ambion) extraction. One microgram of total RNA was subjected to DNase treatment using RQ1 RNase free DNase from Promega. cDNAs were generated using SuperScript III Reverse Transcriptase (Thermo Fisher Scientific) using random hexamers as primers according to manufacturer's instructions. Quantitative PCR was performed using SYBR green mixes and specific primers against tRNA. Levels were normalized to U6snRNA gene. Please see Supplemental Table 1 for list of primers.
For detection of tRFs, $100 \mu \mathrm{g}$ of total RNA was subjected to small RNA enrichment using the miRVANA miRNA Purification Kit (Ambion). Small RNA enriched RNA pool was loaded into $15 \% 7 \mathrm{M}$ urea-polyacrylamide page, and RNAs in the 15-35 nt size range were purified. The size selected RNA was eluted from gel slices overnight in $0.3 \mathrm{M} \mathrm{NaCl} / \mathrm{TE}$ buffer at room temperature and precipitated with 2 volumes of isopropanol $+10 \mu \mathrm{g}$ glycogen at $-20^{\circ} \mathrm{C}$ overnight. RNA was precipitated by centrifugation at $4^{\circ}$ $\mathrm{C}$ for $20 \mathrm{~min}$ at 13,000 rpm and washed once with $70 \% \mathrm{EtOH}$. cDNAs were generated using NCode miRNA First-Strand cDNA Synthesis and qRT-PCR Kits with 50-100 ng 15-35 nt long RNA as starting material. The levels of tRFs are normalized to the levels of miR-21. Please see Supplemental Table 1 for the list of primers.

\section{Northern blotting}

Cells were transfected and collected as described above.

Forty micrograms of TRIzol extracted total RNA was loaded into 15\% $7 \mathrm{M}$ urea-polyacrylamide gels. The RNA was transferred onto Hybond-N+ positively charged nylon membranes (GE Healthcare, cat. no. RPN203B) using a Bio-Rad transblot apparatus at $200 \mathrm{~mA}$ (9-10 V) for $3 \mathrm{~h}$ (Bio-Rad). The transferred RNA was cross-linked to the membrane by UV-irradiation for 1 min (Stratalinker, Stratagene) with 254-nm bulbs with the autocrosslink option. The membrane was dried between two dry $3 \mathrm{M}$ papers by baking at $50^{\circ} \mathrm{C}$ for $30 \mathrm{~min}$. The membrane was stored at $4^{\circ} \mathrm{C}$ between filter papers until use. The rest of the protocol was performed as described in Huang et al. (2014). Briefly, membranes were prehybridized for at least $30 \mathrm{~min}$ at $40^{\circ} \mathrm{C}$ in prehybridization buffer (7\% SDS, $200 \mathrm{mM} \mathrm{Na} \mathrm{HPO}_{4}[\mathrm{pH} \quad 7.0]$ ) containing $5 \mu \mathrm{g} / \mathrm{mL}$ denatured salmon sperm DNA. Hybridization was performed in Expresshyb solution, Clontech cat. no. 636831 containing $50 \mathrm{pmol} / \mathrm{mL}$ labeled probes (Anti-3009: BIO-TGGTACCAGGAGTGGGGT) at $40^{\circ} \mathrm{C}$ overnight (typically $16 \mathrm{~h}$ ). The membrane was washed thrice with $1 \times$ SSC, $0.1 \%$ SDS at RT for 5 min. ECL Lightning was performed using the Chemiluminescent Nucleic Acid Detection Module Kit from Thermo Scientific following the instructions in their manual.

\section{Dual luciferase assay}

Luciferase assays were performed in 24-well plates. Four-hundred eighty nanograms of tRNA overexpression plasmid or empty vector pcDNA3 and 2 ng of psi-CHECK2 reporter vector with no sites or perfect complementary sites to tRF- 3 in the $3^{\prime}$ UTR of Renilla luciferase gene were transfected in to HEK293T cells using Lipofectamine 2000 (Life Technologies) as a transfection reagent. The cells were lysed in $100 \mu \mathrm{L} 1 \times$ passive lysis buffer from the Dual-Luciferase Reporter Assay System (Promega) after $2 \mathrm{~d}$ of transfection. The luciferase signals were measured using $20 \mu \mathrm{L}$ of the lysate following the instructions provided by Promega. The Renilla luciferase levels were normalized to firefly luciferase levels and the results were always plotted with tRF overexpression over nonoverexpression (see figure legends).

Luciferase reporter assays were done in the same way with reporters containing $3^{\prime}$ UTRs of endogenous genes.

\section{siRNA knockdown experiments}

To knock down Argonaute proteins, $20 \mathrm{nM}$ of siControl(Sigma) or $20 \mathrm{nM}$ total of siRNAs against Ago1, 2, and 3 were transfected into 
HEK293T cells using RNAimax transfection reagent from Life Technologies, and this was repeated after $24 \mathrm{~h}$. Luciferase reporters and tRNA overexpression plasmids were transfected $24 \mathrm{~h}$ after second siRNA transfection. Lysates were collected $48 \mathrm{~h}$ after second siRNA transfection for western blot analysis and dual luciferase assay.

\section{Western blotting}

Argonaute protein levels were detected by western blotting. Briefly, the membrane was incubated in 5\% milk in TBS-T blocking solution for $1 \mathrm{~h}$ at room temperature and in rabbit monoclonal primary (Ago1: CST\#5053, Ago2: CST\#2897) in 5\% BSA, TBS-T with 1:1000 dilution for overnight at $4^{\circ} \mathrm{C}$. The membrane was washed three times in 1× TBS-T and incubated with 1:5000 diluted HRP goat-anti-rabbit secondary in 5\% BSA, TBST at room temperature. Immobilon Western Chemiluminescent HRP Substrate from Millipore was used for developing the signal.

Twenty micrograms of cell lysate from wild-type and Dicer knockout cells was loaded into 7.5\% SDS-PAGE gel to detected Dicer protein levels by western blotting. Blocking was performed in 3\% milk in PBS-T for $1 \mathrm{~h}$ at room temperature. 1:1000 dilution of Dicer antibody (Abcam ab14601) and 1:2000 dilution of $\alpha$-tubulin antibody (Santa Cruz sc-5286) was used as a primary at $4^{\circ} \mathrm{C}$ for overnight.

\section{AGO immunoprecipitation}

Immunoprecipitation of Argonaute proteins from tRNA overexpressing cells was performed as described in the MAGNA-RIP Kit (Millipore 17-700) using Pan-Ago antibody (Millipore MABE56). RNA was extracted by phenol chloroform as described in the kit and loaded into 15\% $7 \mathrm{M}$ urea-polyacrylamide gel. Northern was performed as described above.

\section{RNA-seq library preparation}

HEK293T cells were transfected with empty vector or tRNA expression plasmids four times in $2 \mathrm{~d}$ intervals. Total RNA was purified with the Qiagen RNeasy Kit. tRNA and tRF levels were quantified as described above.

One microgram of total RNA from pcDNA3 and tRNA LeuTAA (tRF-3009) overexpressing cells were used for library preparation. Libraries were prepared using the NEBNext Ultra Directional RNA Library Prep Kit with NEBNext Poly(A) mRNA Magnetic Isolation Module for Illumina. The libraries were indexed using NEBNext Multiplex Oligos for Illumina. Quality controls and sequencing were done in the Genomic Services Laboratory at Hudson Alpha.

\section{Analysis of the small RNA data isolated from Dicer knockout and Dicer WT cells}

We analyzed two small RNA data sets isolated from Dicer knockout and wild-type cell lines generated by two independent laboratories (Bogerd et al. 2014; Kim et al. 2016). Firstly, adaptor sequence was removed using the "Cutadapt" program (Martin 2011) and sequencing reads that were $\geq 14$ bases long were retained. To identify the total mapped reads, the small RNA reads were mapped on whole genome (hg38 genome build) by using short read aligner Novoalign (http://www.novocraft.com/products/novoalign/). Next, the identical reads were collapsed and mapped on to the in house built small RNA database (mature miRNA and tRNA as detailed in Kumar et al. 2014, 2015) using BLASTn (Altschul et al. 1990). The building of the in house blast database for blast searches is explained in detail in our earlier publications (Kumar et al. 2014, 2015). The total number of mapped reads was used for normalizing the expression of miRNA and tRFs. In general, we considered only those alignments where the query sequence (small RNA) was mapped to the database sequence (tRNA or miRNA) along $100 \%$ of its length. The blast output file was parsed to get information on the mapped position of small RNA on tRNA or miRNA. We extracted all map positions where the small RNA aligned from its first base to the last base with the tRNA sequence allowing either one or no mismatch. Since "CCA" is added at the $3^{\prime}$ end of tRNA by tRNA nucleotidyltransferase during maturation of tRNA (Xiong and Steitz 2006), we allowed a special exception for the small RNA mapping to the $3^{\prime}$ ends of tRNAs allowing a terminal mismatch of $\leq 3$ bases. To remove any false positives, the small RNAs that mapped on to the "tRNAdb" were again searched against the whole genome using blast search excluding the tRNA loci.

\section{Analysis of PAR CLIP data}

We also investigated tRF and miRNA expression in human small RNA PARCLIP data of TNRC6A, B, and C by analyzing the human TNRC6A (GEO ID = GSM545218), B (GEO ID = GSM545219), and C (GEO ID = GSM545220) PAR-CLIP data isolated from HEK293 cell lines (Hafner et al. 2010). Data from all three small RNA libraries were examined for miRNA and tRF expression as well as for the $\mathrm{T}$ to $\mathrm{C}$ mutation position and its frequency compared to wild-type small RNAs (miRNAs and tRFs). Sequence reads that either mapped perfectly on miRNA or tRFs or mapped with one base mismatch were considered for $\mathrm{T}$ to $\mathrm{C}$ mutation analysis. The mismatched base and its position relative to the $5^{\prime}$ end of small RNA were collected for final analysis.

The 17,319 CCRs identified by Hafner et al. (2010) present in the PAR-CLIP data were used to study the complementary sequence of miRNA and tRF-3 seed sequence along the length of CCR. All the possible 7-mer sequences were generated along the length of miRNA and tRF. The 7-mer sequences were reverse complemented and mapped and the match was scored along the length of CCR. CCRs are $41 \mathrm{nt}$ long sequences centered at the $\mathrm{T}$ (protein binding site) that showed the highest $\mathrm{T}$ to $\mathrm{C}$ frequency. Hafner et al. (2010) demonstrated that the reverse complement of known miRNA seeds is enriched in CCRs directly following this central cross-linked T. In our analysis, four 7-mer (1-7, 2-8, 3-9, and 410) reverse complementary sequences of the top 20 abundant miRNA and tRF-3 sequences identified in TNRC6A-C (a member of GW-bodies or P-bodies) were used for finding sequences along the length of CCR. The counting and scanning of the CCRs was done from the $5^{\prime}$ end to $3^{\prime}$ end of CCRs. Whenever there was a match, the score (count) was assigned to all the seven bases of CCR.

\section{Analysis of RNA-seq data}

We received on average 30 million 50 bases long paired-end reads for each of the replicates and had two replicates for each condition. The transcript (RefSeq genes) sequences for the genome build hg38 
were downloaded from the UCSC Table Browser on Dec 10, 2016 (http://genome.ucsc.edu). We used default parameters of Kallisto (Bray et al. 2016) to build an index for the above transcript sequence and then quantified abundances of the transcripts from the pairedend RNA-seq fastq reads (Bray et al. 2016). The DESeq2 (Love et al. 2014) package in $\mathrm{R}$ was used for differential expression analysis of the quantified data obtained from Kallisto. The normalized count data from DESeq2 were used for all other downstream analysis. The data have been deposited to the Gene Expression Omnibus (GEO) database, www.ncbi.nlm.nih.gov/geo (GSE99769).

\section{tRF target gene prediction}

The 3'UTR sequence of each annotated RefSeq gene was downloaded using the UCSC Table Browser (hg38 genome build). We decrease the noise from the lowly expressed isoform by considering only the most expressed isoform of each gene in Hela cells (Nam et al. 2014) for downstream analysis. A total of 9294 sequences were examined for the complementarity of the tRF-3009 sequence using the default parameter RNA22 (Miranda et al. 2006). A total of $11193^{\prime}$ UTR sequences were identified that had at least a 6-mer complementary sequence to the $5^{\prime}$ region of the tRF-3009 (Miranda et al. 2006). These identified targets were used to compare the expression of target with nontarget genes.

\section{Cumulative distribution function plot (CDF plot) of tRF target and nontarget genes}

Cumulative distribution function of R (ecdf) was used to compare the plot between targets and nontarget genes in various experimental conditions. ks.test (Kolmogrov-Smirnov test), a function in $\mathrm{R}$ package, was used to test if the plot of target genes is above the plot of nontarget genes and if the difference is statistically significant.

\section{SUPPLEMENTAL MATERIAL}

Supplemental material is available for this article.

\section{ACKNOWLEDGMENTS}

We would like to thank all the members of the Dutta laboratory for fruitful discussions. We also would like to thank Dr. Bryan Cullen for the Dicer knockout 293T cells. This work was supported by National Institutes of Health grants P01 CA104106 (Project 3) and R01 GM84465 to A.D. M.K. was partly supported by a US Department of Defense (DOD) award (PC151085).

Author contributions: C.K. and A.D. designed the experiments. C.K. is responsible for all biological experiments and P.K. is responsible for bioinformatics analysis. Initial bioinformatics analysis was done by M.K. A.M. performed Ago western blots. Z.S. performed some of the biological experiments. C.K. and A.D. wrote the paper.

Received February 8, 2018; accepted May 24, 2018.

\section{REFERENCES}

Altschul SF, Gish W, Miller W, Myers EW, Lipman DJ. 1990. Basic local alignment search tool. J Mol Biol 215: 403-410.
Arabi A, Wu S, Ridderstråle K, Bierhoff $\mathrm{H}$, Shiue C, Fatyol K, Fahlén S, Hydbring P, Söderberg O, Grummt I, et al. 2005. c-Myc associates with ribosomal DNA and activates RNA polymerase I transcription. Nat Cell Biol 7: 303-310.

Bernstein E, Caudy AA, Hammond SM, Hannon GJ. 2001. Role for a bidentate ribonuclease in the initiation step of RNA interference. Nature 409: 363-366.

Betancur JG, Tomari Y. 2012. Dicer is dispensable for asymmetric RISC loading in mammals. RNA 18: 24-30.

Bogerd HP, Whisnant AW, Kennedy EM, Flores O, Cullen BR. 2014. Derivation and characterization of Dicer- and microRNA-deficient human cells. RNA 20: 923-937.

Bray NL, Pimentel H, Melsted P, Pachter L. 2016. Near-optimal probabilistic RNA-seq quantification. Nat Biotechnol 34: 525-527.

Brennecke J, Stark A, Russell RB, Cohen SM. 2005. Principles of microRNA-target recognition. PLoS Biol 3: e85.

Cheloufi S, Dos Santos CO, Chong MM, Hannon GJ. 2010. A dicer-independent miRNA biogenesis pathway that requires Ago catalysis. Nature 465: 584-589.

Chen Q, Yan M, Cao Z, Li X, Zhang Y, Shi J, Feng GH, Peng H, Zhang X, Zhang Y, et al. 2016. Sperm tsRNAs contribute to intergenerational inheritance of an acquired metabolic disorder. Science 351: 397-400.

Chendrimada TP, Gregory RI, Kumaraswamy E, Norman J, Cooch N, Nishikura K, Shiekhattar R. 2005. TRBP recruits the Dicer complex to Ago2 for microRNA processing and gene silencing. Nature 436: 740-744.

Cole C, Sobala A, Lu C, Thatcher SR, Bowman A, Brown JW, Green PJ, Barton GJ, Hutvagner G. 2009. Filtering of deep sequencing data reveals the existence of abundant Dicer-dependent small RNAs derived from tRNAs. RNA 15: 2147-2160.

Cozen AE, Quartley E, Holmes AD, Hrabeta-Robinson E, Phizicky EM, Lowe TM. 2015. ARM-seq: AlkB-facilitated RNA methylation sequencing reveals a complex landscape of modified tRNA fragments. Nat Methods 12: 879-884.

Eulalio A, Huntzinger E, Izaurralde E. 2008. GW182 interaction with Argonaute is essential for miRNA-mediated translational repression and mRNA decay. Nat Struct Mol Biol 15: 346-353.

Filipowicz W, Bhattacharyya SN, Sonenberg N. 2008. Mechanisms of post-transcriptional regulation by microRNAs: are the answers in sight? Nat Rev Genet 9: 102-114.

Foulkes WD, Priest JR, Duchaine TF. 2014. DICER1: mutations, microRNAs and mechanisms. Nat Rev Cancer 14: 662-672.

Gomez-Roman N, Grandori C, Eisenman RN, White RJ. 2003. Direct activation of RNA polymerase III transcription by c-Myc. Nature 421: 290-294.

Grandori C, Gomez-Roman N, Felton-Edkins ZA, Ngouenet C, Galloway DA, Eisenman RN, White RJ. 2005. c-Myc binds to human ribosomal DNA and stimulates transcription of rRNA genes by RNA polymerase I. Nat Cell Biol 7: 311-318.

Gregory RI, Chendrimada TP, Cooch N, Shiekhattar R. 2005. Human RISC couples microRNA biogenesis and posttranscriptional gene silencing. Cell 123: 631-640.

Grewal SS. 2015. Why should cancer biologists care about tRNAs? tRNA synthesis, mRNA translation and the control of growth. Biochim Biophys Acta 1849: 898-907.

Grimson A, Farh KK, Johnston WK, Garrett-Engele P, Lim LP, Bartel DP. 2007. MicroRNA targeting specificity in mammals: determinants beyond seed pairing. Mol Cell 27: 91-105.

Hafner M, Landthaler M, Burger L, Khorshid M, Hausser J, Berninger P, Rothballer A, Ascano MJr, Jungkamp AC, Munschauer M, et al. 2010. Transcriptome-wide identification of RNA-binding protein and microRNA target sites by PAR-CLIP. Cell 141: 129-141.

Hansen TB, Venø MT, Jensen TI, Schaefer A, Damgaard CK, Kjems J. 2016. Argonaute-associated short introns are a novel class of gene regulators. Nat Commun 7: 11538.

Haussecker D, Huang Y, Lau A, Parameswaran P, Fire AZ, Kay MA. 2010. Human tRNA-derived small RNAs in the global regulation of RNA silencing. RNA 16: 673-695. 
Helwak A, Kudla G, Dudnakova T, Tollervey D. 2013. Mapping the human miRNA interactome by CLASH reveals frequent noncanonical binding. Cell 153: 654-665.

Honda S, Morichika K, Kirino Y. 2016. Selective amplification and sequencing of cyclic phosphate-containing RNAs by the cP-RNAseq method. Nat Protoc 11: 476-489.

Huang Q, Mao Z, Li S, Hu J, Zhu Y. 2014. A non-radioactive method for small RNA detection by northern blotting. Rice (N Y) 7: 26.

Jonas S, Izaurralde E. 2015. Towards a molecular understanding of microRNA-mediated gene silencing. Nat Rev Genet 16: 421-433.

Keam SP, Hutvagner G. 2015. tRNA-derived fragments (tRFs): emerging new roles for an ancient RNA in the regulation of gene expression. Life (Basel) 5: 1638-1651.

Kim YK, Kim B, Kim VN. 2016. Re-evaluation of the roles of DROSHA, Export in 5, and DICER in microRNA biogenesis. Proc Natl Acad Sci 113: E1881-E1889.

Kim HK, Fuchs G, Wang S, Wei W, Zhang Y, Park H, Roy-Chaudhuri B, $\mathrm{Li} \mathrm{P}, \mathrm{Xu} \mathrm{J}$, Chu K, et al. 2017. A transfer-RNA-derived small RNA regulates ribosome biogenesis. Nature 552: 57-62.

Kumar P, Anaya J, Mudunuri SB, Dutta A. 2014. Meta-analysis of tRNA derived RNA fragments reveals that they are evolutionarily conserved and associate with AGO proteins to recognize specific RNA targets. BMC Biol 12: 78.

Kumar P, Mudunuri SB, Anaya J, Dutta A. 2015. tRFdb: a database for transfer RNA fragments. Nucleic Acids Res 43: D141-D145.

Kumar P, Kuscu C, Dutta A. 2016. Biogenesis and function of transfer RNA-related fragments (tRFs). Trends Biochem Sci 41: 679-689.

Lee YS, Shibata Y, Malhotra A, Dutta A. 2009. A novel class of small RNAs: tRNA-derived RNA fragments (tRFs). Genes Dev 23: 26392649.

Lewis BP, Burge CB, Bartel DP. 2005. Conserved seed pairing, often flanked by adenosines, indicates that thousands of human genes are microRNA targets. Cell 120: 15-20.

Lin CY, Lovén J, Rahl PB, Paranal RM, Burge CB, Bradner JE, Lee TI, Young RA. 2012. Transcriptional amplification in tumor cells with elevated c-Myc. Cell 151: 56-67.

Love MI, Huber W, Anders S. 2014. Moderated estimation of fold change and dispersion for RNA-seq data with DESeq2. Genome Biol 15: 550.

Martin M. 2011. Cutadapt removes adapter sequences from highthroughput sequencing reads. EMBnet.journal 17: 3 .

Maute RL, Schneider C, Sumazin P, Holmes A, Califano A, Basso K, Dalla-Favera R. 2013. tRNA-derived microRNA modulates proliferation and the DNA damage response and is down-regulated in B cell lymphoma. Proc Natl Acad Sci 110: 1404-1409.

Meister G. 2013. Argonaute proteins: functional insights and emerging roles. Nat Rev Genet 14: 447-459.

Miranda KC, Huynh T, Tay Y, Ang YS, Tam WL, Thomson AM, Lim B, Rigoutsos I. 2006. A pattern-based method for the identification of
microRNA binding sites and their corresponding heteroduplexes. Cell 126: 1203-1217.

Nam JW, Rissland OS, Koppstein D, Abreu-Goodger C, Jan CH, Agarwal V, Yildirim MA, Rodriguez A, Bartel DP. 2014. Global analyses of the effect of different cellular contexts on microRNA targeting. Mol Cell 53: 1031-1043.

Nielsen CB, Shomron N, Sandberg R, Hornstein E, Kitzman J, Burge CB. 2007. Determinants of targeting by endogenous and exogenous microRNAs and siRNAs. RNA 13: 1894-1910.

Pavon-Eternod M, Gomes S, Geslain R, Dai Q, Rosner MR, Pan T. 2009. tRNA over-expression in breast cancer and functional consequences. Nucleic Acids Res 37: 7268-7280.

Pliatsika V, Loher P, Magee R, Telonis AG, Londin E, Shigematsu M, Kirino Y, Rigoutsos I. 2018. MINTbase v2.0: a comprehensive database for tRNA-derived fragments that includes nuclear and mitochondrial fragments from all The Cancer Genome Atlas projects. Nucleic Acids Res 46: D152-D159.

Saikia M, Hatzoglou M. 2015. The many virtues of tRNA-derived stressinduced RNAs (tiRNAs): discovering novel mechanisms of stress response and effect on human health. J Biol Chem 290: 29761-29768.

Schopman NC, Heynen S, Haasnoot J, Berkhout B. 2010. A miRNAtRNA mix-up: tRNA origin of proposed miRNA. RNA Biol 7: 573-576.

Schorn AJ, Gutbrod MJ, LeBlanc C, Martienssen R. 2017. LTR-retrotransposon control by tRNA-derived small RNAs. Cell 170: 61-71 el1.

Selitsky SR, Sethupathy P. 2015. tDRmapper: challenges and solutions to mapping, naming, and quantifying tRNA-derived RNAs from human small RNA-sequencing data. BMC Bioinformatics 16: 354.

Sharma U, Conine CC, Shea JM, Boskovic A, Derr AG, Bing XY, Belleannee C, Kucukural A, Serra RW, Sun F, et al. 2016. Biogenesis and function of tRNA fragments during sperm maturation and fertilization in mammals. Science 351: 391-396.

Telonis AG, Loher P, Honda S, Jing Y, Palazzo J, Kirino Y, Rigoutsos I. 2015. Dissecting tRNA-derived fragment complexities using personalized transcriptomes reveals novel fragment classes and unexpected dependencies. Oncotarget 6: 24797-24822.

Thompson A, Zielezinski A, Plewka P, Szymanski M, Nuc P, Szweykowska-Kulinska Z, Jarmolowski A, Karlowski WM. 2018. tRex: a web portal for exploration of tRNA-derived fragments in Arabidopsis thaliana. Plant Cell Physiol 59: e1.

Xiong Y, Steitz TA. 2006. A story with a good ending: tRNA 3'-end maturation by CCA-adding enzymes. Curr Opin Struct Biol 16: 12-17.

Zheng G, Qin Y, Clark WC, Dai Q, Yi C, He C, Lambowitz AM, Pan T. 2015. Efficient and quantitative high-throughput tRNA sequencing. Nat Methods 12: 835-837.

Zheng LL, Xu WL, Liu S, Sun WJ, Li JH, Wu J, Yang JH, Qu LH. 2016. tRF2Cancer: a web server to detect tRNA-derived small RNA fragments (tRFs) and their expression in multiple cancers. Nucleic Acids Res 44: W185-W193. 

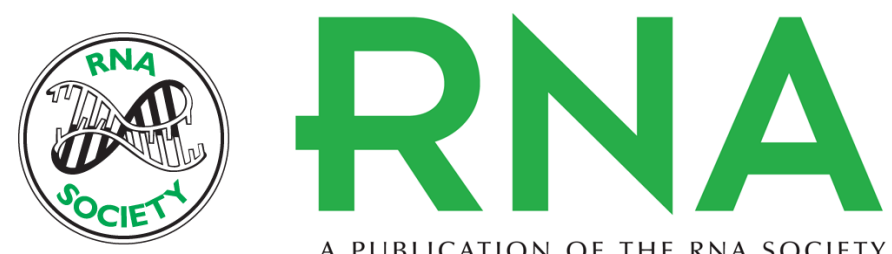

A PUBLICATION OF THE RNA SOCIETY

\section{tRNA fragments (tRFs) guide Ago to regulate gene expression post-transcriptionally in a Dicer-independent manner}

Canan Kuscu, Pankaj Kumar, Manjari Kiran, et al.

RNA 2018 24: 1093-1105 originally published online May 29, 2018

Access the most recent version at doi:10.1261/rna.066126.118

\section{Supplemental http://rnajournal.cshlp.org/content/suppl/2018/05/29/rna.066126.118.DC1 \\ Material}

References This article cites 55 articles, 11 of which can be accessed free at: http://rnajournal.cshlp.org/content/24/8/1093.full.html\#ref-list-1

Creative This article is distributed exclusively by the RNA Society for the first 12 months after the Commons License full-issue publication date (see http://rnajournal.cshlp.org/site/misc/terms.xhtml). After 12 months, it is available under a Creative Commons License (Attribution-NonCommercial 4.0 International), as described at http://creativecommons.org/licenses/by-nc/4.0/.
Email Alerting Receive free email alerts when new articles cite this article - sign up in the box at the Service top right corner of the article or click here.

\section{IIII!" Providing Precise Solutions for your research.}

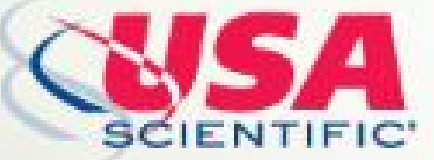

To subscribe to $R N A$ go to:

http://rnajournal.cshlp.org/subscriptions 